\title{
Post-starburst galaxies and the transformation of blue into red galaxies
}

\author{
S. De Rijcke ${ }^{1, \star}$, P. Buyle ${ }^{1}$, D.J. Pisano ${ }^{2}$, K. Freeman ${ }^{3}$, and H. Dejonghe ${ }^{1}$ \\ 1 Astronomical Observatory, Ghent University, Krijgslaan 281, S9, B-9000 Gent, Belgium \\ 2 NRAO, P.O. Box 2 Green Bank, WV 24944, USA \\ 3 Research School of Astronomy \& Astrophysics, Mount Stromlo Observatory, Cotter Road, Weston ACT 2611, Australia
}

Received 2009 Aug 14, accepted 2009 Sep 14

Published online 2009 Oct 20

\begin{abstract}
Key words galaxies: elliptical and Lenticular, $\mathrm{cD}$ - galaxies: evolution - galaxies: fundamental parameters - galaxies: ISM

We present deep single-dish radio observations of a sample of nearby post-starburst galaxies $(0.05<z<0.1)$. About $50 \%$ of these post-starburst galaxies are detected at $21 \mathrm{~cm}$, with $\mathrm{H}$ I masses of $\sim 10^{9} \mathrm{M}_{\odot}$, up to $\sim 10^{10} \mathrm{M}_{\odot}$. These post-starburst galaxies are as gas-rich as spiral galaxies with comparable luminosities. There appears to exist no direct correlation between the amount of $\mathrm{H}$ I present in a post-starburst galaxy and its star formation rate as traced by radio continuum emission. Moreover, the end of the starburst clearly does not necessarily require the complete exhaustion of the neutral gas reservoir. High-resolution radio observations of one post-starburst binary system suggest that most of the neutral gas resides outside the stellar bodies of the galaxies. Most likely, the gas was expelled by supernova and/or AGN feedback. This effectively stops star formation, even though copious amounts of diffuse neutral gas remain in the immediate vicinity. This remaining $\mathrm{H}$ I reservoir may eventually lead to further episodes of star formation. This may indicate that some post-starbursts are observed in the inactive phase of the star formation duty cycle.
\end{abstract}

(C) 2009 WILEY-VCH Verlag GmbH \& Co. KGaA, Weinheim

\section{Introduction}

Poststarburst galaxies (or PSGs, or E+A galaxies, or k+a/$\mathrm{a}+\mathrm{k}$ galaxies) are characterized by their optical spectra, independent of any morphological or photometric considerations. PSGs contain a sizable population of very young stars, producing very prominent Balmer lines, but lack ongoing star formation, hence the absence of detectable emission lines such as [O II] $\lambda 3727$. This suggests that PSGs are observed very shortly, within less than $1 \mathrm{Gyr}$, after the end of a vigorous, abruptly truncated starburst phase (Dressler \& Gunn 1983; Dressler et al. 1999; Poggianti et al. 1999).

Today, they constitute only a small fraction of the galaxy cluster population (less than 1\%; Fabricant et al. 1991); at intermediate redshifts, however, they constitute a substantial cluster population (Belloni et al. 1995). They span the whole morphological range, from bulge-dominated with underlying disks to disk-dominated (Tran et al. 2003; Yang et al. 2004). Their high surface brightness sets them apart from the elliptical and lenticular galaxies in the fundamental plane. Over time, fading of the stellar population will drive them toward the locus of the E/S0 galaxies (Yang et al. 2004; Poggianti et al. 1999). Internal velocity dispersions of galaxies classified as PSGs appear to increase as a function of redshift, going from $\sigma \sim 150 \mathrm{~km} \mathrm{~s}^{-1}$ at $z=0.3$ to $\sigma \sim 250 \mathrm{~km} \mathrm{~s}^{-1}$ at $z=0.83$ (Tran et al. 2003). This trend suggests that massive galaxies undergo a PSG phase, i.e., are observed in a poststarburst phase, at earlier cosmic times

\footnotetext{
^ Corresponding author: sven.derijcke@UGent.be
}

than less massive ones. This is reminiscent of the "downsizing” phenomenon in star-forming galaxies (Cowie et al. 1996), according to which the masses of galaxies hosting star formation decrease as the universe ages.

Although the majority of low- $z$ PSG galaxies have a smooth light distribution, many of them also show slightly disturbed morphologies (e.g., warps and dust lanes). Based on this, Zabludoff et al. (1996) argued that the PSG phase is the aftermath of a vigorous starburst, triggered by a major merger or interaction. This is corroborated by a study of PSG galaxies drawn from the Two Degree Field Galaxy Redshift Survey (2dFGRS) catalog (Blake et al. 2004). About three quarters of all PSG galaxies are found in the field (i.e., outside clusters), simply because most of the galaxies in the universe do not reside in clusters. However, the fraction of PSGs is 4 times higher in clusters than in the field (Zabludoff et al. 1996; Tran et al. 2004). The spatial distribution of PSG galaxies in clusters is more extended than that of quiescent galaxies, but less extended than that of emission-line galaxies (Dressler et al. 1999), suggesting that processes such as galaxy harassment or ram pressure stripping, which are specific to clusters, can also cause the PSG phenomenon. Statistical studies of the environments of PSGs (Blake et al. 2004; Goto 2005; Yamauchi et al. 2008) have shown that the distribution of local environments of PSG galaxies closely traces that of the ensemble of galaxies but that they have a significantly higher probability of having close companions. These authors conclude that whatever causes the PSG phenomenon, must be a very local mechanism, such as encounters of galaxy pairs. 
Most PSG galaxies have E/S0-like morphologies, with a small fraction of ongoing interactions. Their luminosity distribution is more similar to the distribution of spectroscopically defined elliptical galaxies than to the luminosity distribution of the ensemble of 2dFGRS galaxies (Blake et al. 2004). However, not all PSGs can be associated with mergers and, obviously, more than one evolutionary pathway can lead to a post-starburst galaxy (Tran et al. 2003; Dressler et al. 1999). Numerical simulations show that PSGs can indeed be formed via a major merger of two gas-rich spiral galaxies (Bekki et al. 2005). A disk-disk merger event then triggers a starburst, which consumes, or, by feedback, expels most of the available gas and then subsides. The young stars then dominate the optical spectrum for the following few hundred megayears while emission lines are absent. During this time span, a galaxy would be classified as an PSG. In this case, one expects star formation to be centrally concentrated, leading to radially decreasing Balmer line strengths (Pracy et al. 2005). Alternatively, star formation can be truncated more or less instantaneously over the whole disk of the galaxy without a starburst, e.g., by the gas being swept away by ram pressure stripping. In this case, as the young star population fades, the older bulge population causes the strengths of the Balmer lines to be radially increasing (Pracy et al. 2005).

The red colors of some $\mathrm{H} \delta$-strong PSG galaxies cannot be explained by any plausible starburst model (Couch \& Sharples 1987; Blake et al. 2004), leaving heavy dust extinction as the only viable explanation. This hypothesis can be tested by using dust insensitive tracers of star formation. Since radio continuum emission is synchrotron radiation from electrons accelerated in supernova remnants, it is an indirect tracer of star formation. Miller \& Owen (2001) observed part of the Zabludoff et al. (1996) sample and detected radio continuum emission in only two out of 15 galaxies. Smail et al. (1999) detected five out of eight poststarburst galaxies at radio wavelengths. Radio continuum observations of a sample of 36 PSGs drawn from the SDSS yielded no detections (Goto 2004). If this apparent lack of ongoing star formation in PSGs is true, then no dust obscuration, hiding the star formation sites, needs to be invoked. Near-infrared studies (Galaz 2000; Balogh et al. 2005) have shown that the $u-g$ and $r-k$ colors, and the $\mathrm{H} \delta$ line strengths of PSGs, can be well explained by dust-free models in which more than $5 \%$ of the stellar mass has recently been produced in a starburst. Hence, the presence of dust is still uncertain because of these contradictory observations.

In the nearby universe, galaxies have a bimodal color distribution, with a blue peak of star-forming galaxies separated from a red sequence of quiescent galaxies by a sparsely populated gap (Strateva et al. 2001; Baldry et al. 2004; Balogh et al. 2004). The number of red galaxies has roughly doubled since $z \sim 1$ (Bell et al. 2004). Red galaxies, on average, are more luminous than blue galaxies. Therefore, a simple cessation of star formation in a fraction of the blue galaxies followed by fading and reddening cannot explain this buildup of the red sequence. Starbursts are often suggested as a way of increasing a blue galaxy's stellar mass before letting it fade onto the red sequence (Bell et al. 2004; Labbé et al. 2007), with mergers and interactions as possible starburst triggers (Bekki et al. 2005; Di Matteo et al. 2008). Mergers and starbursts have the additional appeal that they offer an explanation for the cessation of star formation: gas is being consumed by the starburst (Di Matteo et al. 2007) and is being expelled by active galactic nucleus (AGN) or supernova feedback (Buyle et al. 2008; Kaviraj et al. 2007). Thus, previously blue galaxies turn red and, through further dry mergers, can lead to the formation of the most massive elliptical galaxies observed in the nearby universe (Bell et al. 2006; Naab et al. 2006). This prompted us to investigate the crucial but transient poststarburst phase, during which a galaxy crosses the color gap, of this particular evolutionary pathway.

PSGs offer us a unique window on how starbursts affect galaxy evolution. The starburst population is fading but still quite prominent and we can study both the burst and the underlying intermediate-age and/or old stellar populations. Due to their high surface brightness, we can study their stellar kinematics out to several half-light radii. Clearly, the poststarburst phase is of crucial importance to furthering our understanding of the photometric evolution of the galaxy population.

\section{H I observations of PSGs}

We have performed deep $21 \mathrm{~cm}$ observations of a sample of 20 nearby PSGs using the $64 \mathrm{~m}$ Australia Telescope National Facility (ATNF) Parkes Radio Telescope and the 305 m Arecibo Radio Telescope (Puerto Rico). This sample was compiled from the larger samples of PSGs of Zabludoff et al. (1996) and Goto (2003). The former defined PSGs spectroscopically as galaxies with a mean equivalent width of the $\mathrm{H} \delta, \mathrm{H} \gamma, \mathrm{H} \beta$ Balmer lines larger than $5.5 \AA$ and that of the [O II] emission line larger than $2.5 \AA$ (with emission lines having negative equivalent widths and absorption line positive ones), while the latter uses $\operatorname{EW}(\mathrm{H} \delta)$ greater than $5.5 \AA$ and no [O II] and $\mathrm{H} \alpha$ emission as criteria. Follow-up radio continuum observations by Miller \& Owen (2001) of a subsample of the Zabludoff et al. (1996) sample yielded no detections of significant star formation. Thus, these galaxies should be secure PSGs. For more details about the data reduction and analysis, we refer to Buyle et al. (2006).

These 20 PSGs reside at redshifts $z \sim 0.06-0.12$ (observing PSGs at high redshifts would result in unworkably long integration times). They are much closer to us than the blue galaxies investigated by Labbé et al. (2007). However, we are only interested in the general principle: can starbursts end without consuming all the available gas? If relatively nearby PSGs are able to retain sizable amounts of gas, this will a fortiori be the case also for PGSs at higher redshifts. Moreover, statistical analysis of the environments of PSGs 

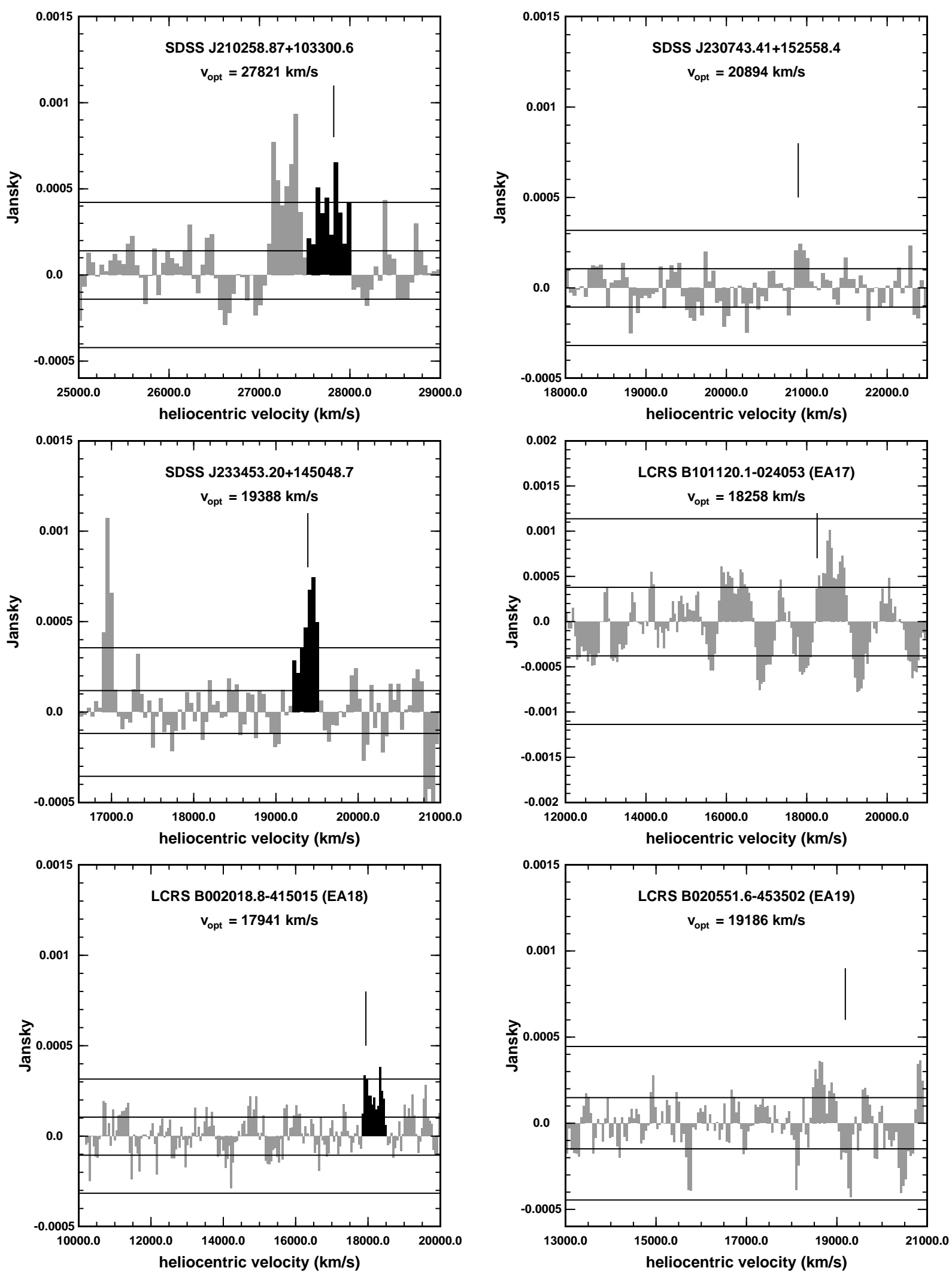

Fig. 1 The H I spectra of our E+As. The galaxies observed with Arecibo, SDSS J210258.87+103300.6, SDSS J2334543.20+145048.7, SDSS J2330743.41+152558.4, have been rebinned to a velocity resolution of $50 \mathrm{~km} \mathrm{~s}^{-1}$. The Parkes dataset, LCRS B101120.1-024053 (EA17), LCRS B002018.8-415015 (EA18), LCRS B020551.6-453502 (EA19), has been rebinnned to a velocity resolution of 53 km s ${ }^{-1}$. The name of the galaxy is indicated in each panel along with its optical systemic velocity. The horizontal black lines indicate the $-3,-1$, 1 and 3 sigma rms noise levels. The vertical black line indicates the optical velocity as found in Goto et al. (2003) and Zabludoff et al. (1996). For the three galaxies with clear detections, the bins corresponding to the galaxy are indicated in black. There is another galaxy within the beam of SDSS J210258.87+103300.6 (the double-horn profile around $27300 \mathrm{~km} \mathrm{~s}^{-1}$ ). 


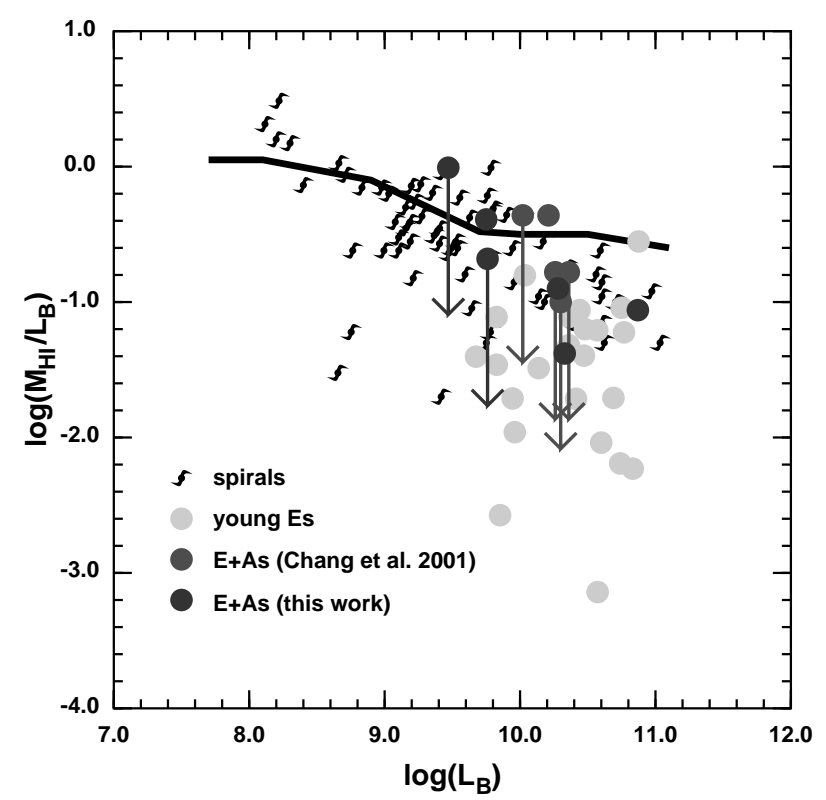

Fig. 2 Plotted are $\log \left(M_{\mathrm{H} \mathrm{I}} / L_{B}\right)$ versus $\log \left(L_{B}\right)$. Spiral galaxies (Helmboldt et al. 2004) are depicted by spiral symbols, elliptical galaxies (Georgakakis et al. 2001) by light grey circles, and post-starburst galaxies by dark grey circles (this work and Chang et al. 2001). The predicted trend for late-type galaxies by the semi-analytical models of Nagashima \& Yoshii (2004) is plotted in black.

and normal galaxies have shown that whatever caused the starburst must be something very local to the galaxy, such as interactions or mergers with immediate neighbours.

We detect 21,cm line emission at more than the $3 \sigma$ significance level in roughly $50 \%$ of the observed PSGs (see, e.g., Fig. 1). This is a much higher detection rate than that reported by Chang et al. (2001), who detect only 1 out of 5 PSGs using the VLA. These galaxies contain more than $10^{9} \mathrm{M}_{\odot}$ of neutral-hydrogen gas, the raw material for future star formation. These post-starburst galaxies are as gasrich as spiral galaxies with comparable luminosities (see Fig. 2). These findings support the idea of episodic star formation from Labbé et al. (2007) who suggest that galaxies undergo periods of strongly enhanced star formation, during which they are blue, followed by periods of quiescence, during which they become redder. This implies that the model galaxies cross the color gap more than once and in both directions. This scenario provides the best quantitative fit to the high- $z$ galaxy color distribution. We have now shown that it is indeed physically plausible that galaxies go through a phase of strongly enhanced star formation and are still left with sufficient amounts of gas for future star formation.

\section{EA01A/B: a binary PSG}

Both members of the EA01A/B binary have a PSG-type optical spectrum and have the same recession velocity. EA01A is the bluest galaxy of the PSG sample of Zabludoff et al.
(1996), suggesting that it is also the youngest sample member. HST/WFPC2 images in the F435W and F702W bands (Yang et al. 2004) provide strong evidence that the couple is interacting. EA01A, positioned about $11^{\prime \prime}$ East of EA01B, contains many stellar clusters that are very conspicuous, compared with the overall rather low surface brightness of this galaxy. This diffuse appearance suggests that it is on the verge of disintegrating due to the injected orbital energy. EA01B, on the other hand, is a bulge-dominated early-type spiral galaxy; it sports strongly asymmetric stellar arms. Both galaxies appear to be connected by a stellar bridge. Previous Hi observations (Chang et al. 2001) had already uncovered sizable quantities of neutral gas in or near these galaxies, but they lacked the spatial resolution to locate the gas with any precision within this galactic binary system.

We retrieved deep, high-resolution H I 21 cm observations of EA01A/B from the science archive of the National Radio Astronomy Observatory (NRAO), made with the Very Large Array (VLA) in New Mexico (USA) (project AM0678). The observations were made with the B configuration during the nights of 2001 March 11, 12, 14, 16, and 18. At a central frequency of $1321.76 \mathrm{MHz}$ ( $\mathrm{L}$ band) and with a bandwidth of $3.125 \mathrm{MHz}$ divided over 63 channels, the $\mathrm{H}$ I in EA01A/B was mapped with a velocity resolution of $11.1 \mathrm{~km} \mathrm{~s}^{-1}$. Alternating observations of the phase calibrator 1127-145 with a 4 minute exposure time and EA01A/B with an exposure time of 25 minutes were made, followed at the end of each night by a 2.5 minute exposure of the flux calibrator 3C 286. This resulted in a total on-source integration time of 32.6 hours. Standard flagging and calibration of the $u-v$ data was performed with the Astronomical Image Processing Software (AIPS). The continuum was subtracted by making a linear fit to the visibilities over the line-free channels that were not affected by the edge effects of the band. We created the final data cube using natural weighting to optimize the sensitivity. This yields a beam size of $8.87^{\prime \prime} \times 6.32^{\prime \prime}$ or $12.3 \times 8.7 h^{-1} \mathrm{kpc}$ at the angular-size distance of EA1 $\left(D_{\mathrm{A}}=285 h^{-1} \mathrm{Mpc}\right)$.

We find a total integrated flux of $S_{\nu}=0.26 \pm 0.03$ $\mathrm{Jy} \mathrm{km} \mathrm{s}{ }^{-1}$. This is equivalent to an $\mathrm{HI}$ mass of $M_{\mathrm{HI}}=$ $(6.6 \pm 0.9) \times 10^{9} \mathrm{M}_{\odot}$, adopting a luminosity distance $D_{L}=330$ Mpc. A plausible interpretation of the H I fluxintegrated map and the channel maps (see Fig. 3) is that we are seeing one receding gaseous tidal arm emanating from EA01A and two tidal arms connected to EA01B, with the east arm approaching us and the west arm moving away from us. Little of the detected gas appears to be directly connected with the galaxies themselves, which show up as minima in the $\mathrm{H}$ I column density. This absence of dense gas may be related to the fact that these galaxies are in a poststarburst phase. The EA01A arm runs in a southeastern direction and can be traced through three consecutive channel maps from 22372.3 to $22394.5 \mathrm{~km} \mathrm{~s}^{-1}$. The west EA01B arm shows up as emission in three consecutive channel maps from 22372.3 to $22394.5 \mathrm{~km} \mathrm{~s}^{-1}$. Following its position through adjacent channel maps with recession 


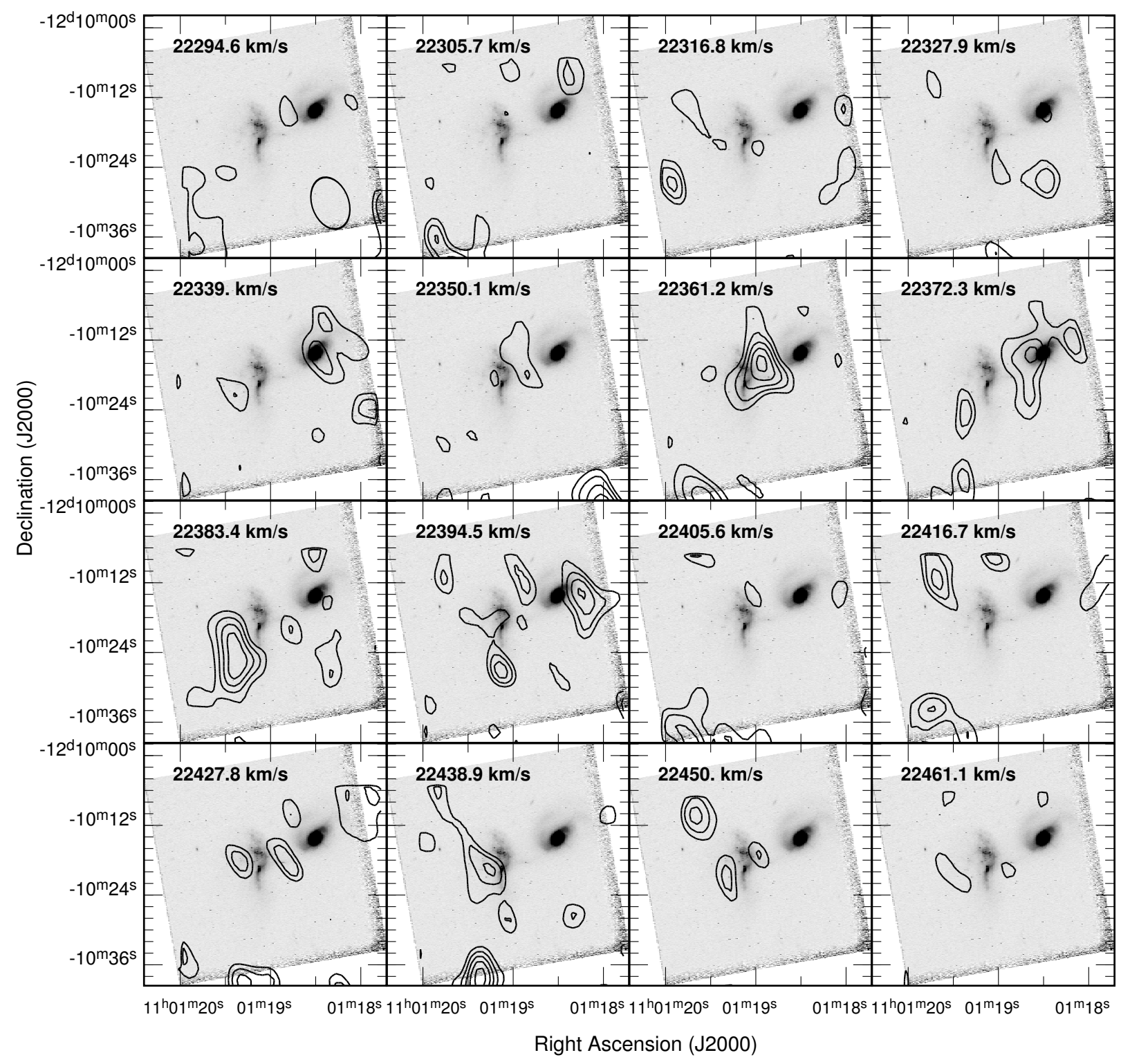

Fig. 3 Channel maps overplotted on an optical HST/WFPC2 F702W image of EA01A/B. The contour levels are $2 \sigma, 3 \sigma, \ldots$ with $\sigma=0.2 \mathrm{mJy}$ beam $^{-1}$. The synthesized beam is depicted in the bottom-right corner of the first map (top left).

velocities below $\approx 22370 \mathrm{~km} \mathrm{~s}^{-1}$, the east arm connected with EA01B appears to first shift in the direction of EA01A and then to return a position slightly north of EA01B before disappearing in the noise. At that point, the gas is moving toward us with a radial velocity of about $40 \mathrm{~km} \mathrm{~s}^{-1}$, relative to the systemic velocity of EA01A/B. The curvature of the northern arm and the fact that it is approaching us agrees with the curvature of the stellar arms.

The galaxies themselves show up as minima in the $\mathrm{HI}$ column density. This lack of galaxy-bound dense neutral gas is most likely connected with the fact that these galaxies are in a poststarburst phase. Supernova and/or AGN feedback are plausible mechanisms that acted to expell the gas from these galaxies.
Acknowledgements. SDR and PB are postdoctoral fellows with the Fund for Scientific Research Flanders (Belgium) (FWO).

\section{References}

Baldry, I.K., et al.: 2004, ApJ 600, 681

Balogh, M.L., et al.: 2004, ApJ 615, L101

Balogh, M.L., Miller, C., Nichol, R., Zabludoff, A., Goto, T.: 2005, MNRAS 360, 587

Bekki, K., Couch, W.J., Shioya, Y., Vazdekis, A.: 2005, MNRAS 359, 949

Bell, E.F., et al.: 2004, ApJ 608, 752

Bell, E., Naab, T., McIntosh, D.H.: 2006, ApJ 640, 241

Belloni, P., Bruzual, A.G., Thimm, G.J., Roser, H.-J.: 1995, A\&A 297, 61 
Blake, C., et al.: 2004, MNRAS 355, 713

Buyle, P., Michielsen, D., De Rijcke, S., Pisano, D.J., Dejonghe, H., Freeman, K.: 2006, ApJ 649, 163

Buyle, P., De Rijcke, S., Dejonghe, H.: 2008, ApJ 684, L17

Chang, T., van Gorkom, J.H., Zabludoff, A., Zaritsky, D., Mihos, C.: 2001, AJ 121, 196

Couch, W.J., Sharples, R.M.: 1987, MNRAS 229, 423

Cowie, L.L., Songaila, A., Hu, E.M., Cohen, J.G.: 1996, AJ 112, 839

Di Matteo, P., Combes, F., Melchior, A.-L., Semelin, B.: 2007, A\&A 468, 61

Di Matteo, P., et al.: 2008, A\&A 492, 31

Dressler, A., Gunn, J.E.: 1983, ApJ 270, 7

Dressler, A., Smail, I., Poggianti, B.M., Butcher, H., Couch, W.J., Ellis, R.S., Oemler, A., Jr.: 1999, ApJS 122, 51

Fabricant, D.G., McClintock, J.E., Bautz, M.W.: 1991, ApJ 381, 33

Galaz, G.: 2000, AJ 119, 2118

Georgakakis, A., Hopkins, A.M., Caulton, A., Wiklind, T., Terlevich, A.I., Forbes, D.A.: 2001, MNRAS 326, 1431

Goto, T.: 2004, A\&A 427, 125

Goto, T.: 2005, MNRAS 357, 937

Goto T., et al.: 2003, PASJ 55, 771

Helmboldt, J.F.: 2007, MNRAS 379, 1227
Helmboldt, J.F., Walterbos, R.A.M., Bothun, G.D., O’Neil, K., de Blok, W.J.G.: 2004, ApJ 613, 914

Kaviraj, S., Kirkby, L.A., Silk, J., Sarzi, M.: 2007, MNRAS 382, 960

Labbé, I., et al.: 2007, ApJ 665, 944

Miller, N.A., Owen, F.N.: 2001, ApJ 554, L25

Naab, T., Khochfar, S., Burkert, A.: 2006, ApJ 636, L81

Nagashima, M., Yoshii, Y.: 2004, ApJ 610, 23

Poggianti, B.M., Smail, I., Dressler, A., et al.: 1999, ApJ 518, 576

Pracy, M.B., Couch, W.J., Blake, C., et al.: 2005, MNRAS 359, 1421

Smail, I., et al.: 1999, ApJ 525, 609

Strateva, I., et al.: 2001, AJ 122, 1861

Tran, K.-V.H., Franx, M., Illingworth, G., Kelson, D.D., van Dokkum, P.: 2003, ApJ 599, 865

Tran, K.-V.H., Franx, M., Illingworth, G.D., van Dokkum, P., Kelson, D.D., Magee, D.: 2004, ApJ 609, 683

Yamauchi, C., Yagi, M., Goto, T.: 2008, MNRAS 390, 383

Yang, Y., Zabludoff, A.I., Zaritsky, D., Lauer, T.R., Mihos, J.C.: 2004, ApJ 607, 258

Yang, Y., Zabludoff, A.I., Zaritsky, D., Mihos, J.C.: 2008, ApJ 688, 945

Zabludoff, A.I., Zaritsky, D., Lin, H., et al.: 1996, ApJ 466, 104 